\title{
Temperature driven air flow through wine corks.
}

\author{
Steven I. Barry ${ }^{1}$
}

(Received 13 August 2008; revised 12 November 2008)

\begin{abstract}
Temperature changes during transport can cause significant air pressure increases within a wine bottle and subsequent loss of air through the cork. With simple modelling this gives rise to a time dependent logistic equation for the air mass. Various solution methods are shown and compared, giving rise to a surprisingly rich mathematical problem which is suitable for university undergraduate courses as an example of an unusual logistic equation. The solutions indicate the importance of the head space in the wine bottle, and give upper bounds on the air flow due to diurnal temperature changes.
\end{abstract}

\section{Contents}

\section{Introduction}

http://anziamj . austms.org. au/ojs/index.php/ANZIAMJ/article/view/1427 gives this article, (c) Austral. Mathematical Soc. 2008. Published December 1, 2008. ISSN 1446-8735. (Print two pages per sheet of paper.) 
2 Modelling

3 Estimations

4 Approximate solutions

5 Results

6 Conclusions

\section{Introduction}

When wine is transported it may be subject to significant temperature variations which affect the quality of the wine. A recent Mathematics in Industry Study Group [4] studied various aspects of this problem, including temperature dependent chemical reactions and thermal transport through containers and arrays of bottles. Work was also done to quantify the amount of air lost through the cork by temperature induced pressure changes. During high temperatures this air loss will include loss of volatiles within the wine, with low temperatures allowing dry air into the bottle. I use the term 'cork' to stand for a variety of bottle closures, including screw tops and synthetic corks. While there has been one small study on the effect of transport on wine [3], there has been no work done on temperature induced air flow through the cork, and no data is available on any air flow through cork.

Figure 1 shows a schematic of the situation with the wine volume increasing and an induced flux, $w(t)$, through the cork.

The purpose of this current study is two fold. First, a model is developed for the loss of air mass through the cork and find solution methods which allow greater understanding of the fundamental behaviour. Second, I illustrate 


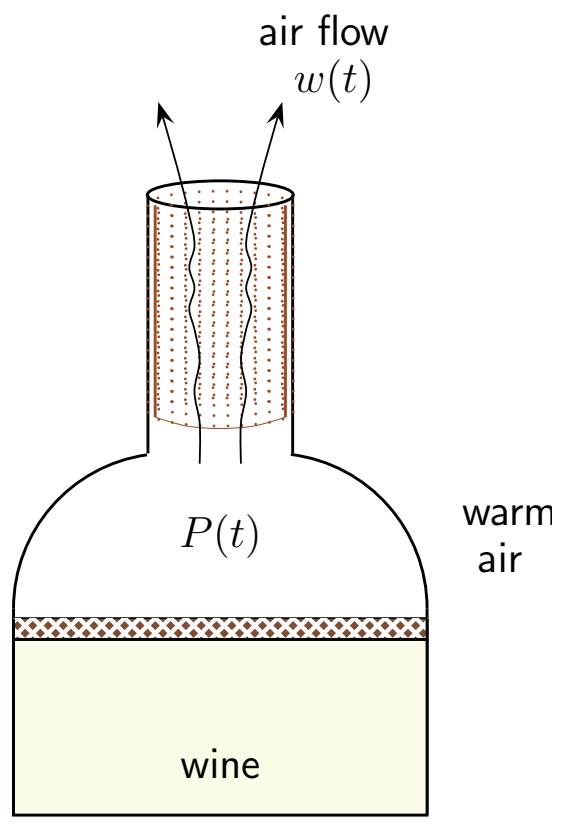

Figure 1: Diagram of a wine bottle showing wine expansion, head space, and air flow through the cork. 
how, with some basic physical principles, a surprisingly rich mathematical problem is found, illustrating many concepts taught to mathematics students; in this way this problem can serve as a useful undergraduate teaching tool.

\section{Modelling}

In this section I develop the model for the amount of air between the wine and the cork. This assumes the bottle is stored vertically as shown in Figure 1. The model is based on three physical principles: the perfect gas law for the air within the bottle; Darcy's law [1] for air flow through the cork, treated as a porous material; and the volumetric expansion coefficient of the liquid.

Darcy's law relates fluid velocity to an applied pressure difference so that

$$
w(t)=k \frac{P(t)-P_{0}}{L},
$$

where $w(t)[\mathrm{m} / \mathrm{s}]$ is the air velocity through the cork at time $\mathrm{t}[\mathrm{s}], \mathrm{P}(\mathrm{t})[\mathrm{Pa}]$ is the pressure, $P_{0}$ is atmospheric pressure assumed to be the same as $P(0)$, $k(t)\left[\mathrm{m}^{2} / \mathrm{s} / \mathrm{Pa}\right]$ is the permeability including the viscosity, and $L[\mathrm{~m}]$ is the length of the cork. The rate of change of air mass through the cork is then

$$
\frac{\mathrm{dm}(\mathrm{t})}{\mathrm{dt}}=-\rho(\mathrm{t}) w(\mathrm{t}) \mathrm{A}=-\mathrm{kA} \frac{\rho(\mathrm{t})\left(\mathrm{P}(\mathrm{t})-\mathrm{P}_{0}\right)}{\mathrm{L}},
$$

where $m(t)[\mathrm{kg}]$ is the mass of air in the gap between cork and wine, $A\left[\mathrm{~m}^{2}\right]$ the cross-sectional area of the cork, and $\rho(t)\left[\mathrm{kg} / \mathrm{m}^{3}\right]$ is the density of the air.

The pressure in the air is modelled by the perfect gas law:

$$
\frac{\mathrm{P}(\mathrm{t}) \mathrm{V}(\mathrm{t})}{\mathrm{m}(\mathrm{t}) \mathrm{T}(\mathrm{t})}=\frac{\mathrm{P}(0) \mathrm{V}(0)}{\mathrm{m}(0) \mathrm{T}(0)} \equiv \alpha
$$


where $T(t)[K]$ is the temperature of the air, $V(t)\left[\mathrm{m}^{3}\right]$ is the volume of air, and $\alpha$ is a constant.

Combining Equations (2) and (3) gives

$$
\frac{d m(t)}{d t}=-\frac{k(t) A}{L}\left[\frac{\alpha m^{2}(t) T(t)}{V^{2}(t)}-\frac{P_{0} m(t)}{V(t)}\right] .
$$

The volume of wine in the bottle is also temperature dependent:

$$
\mathrm{V}_{w}(\mathrm{t})=\mathrm{V}_{w}(0)\left\{1+\beta\left[\mathrm{T}_{w}(\mathrm{t})-\mathrm{T}_{w}(0)\right]\right\},
$$

where $V_{w}(t)$ is the volume of the wine, $m_{w}$ is the mass of wine, $T_{w}(t)$ is the wine temperature, and $\beta(T)[/ K]$ is the volumetric expansion coefficient of wine. The volume of the bottle, $V_{b}$, remains constant hence the air volume is $V_{a}(t)=V_{b}-V_{w}(t)$.

There are other effects which may be included, but are considered to be negligible. The permeability, $k$, will be a function of time. When moist air is flowing out of the bottle, the viscosity will be higher and hence the permeability lower. When dry air flows into the wine the permeability will be higher. The level of moisture in the air will also be temperature dependent as vapour pressures of key components also change [2]. I also ignore some complex thermodynamics of saturated gas/liquid interactions.

While possible to model the temperature of the air and wine separately, I assume here $\mathrm{T}_{w}(\mathrm{t}) \approx \mathrm{T}(\mathrm{t})$. Future models will include temperature effects within the glass, the wine and the air.

This problem is simplified by writing

$$
\begin{aligned}
& m(t)=m(0) m^{*}\left(t^{*}\right), \quad V(t)=V(0) V^{*}\left(t^{*}\right), \\
& P(t)=P(0) P^{*}\left(t^{*}\right), \quad k(t)=k(0) k^{*}\left(t^{*}\right), \quad t=t_{0} t^{*},
\end{aligned}
$$

where * denotes non-dimensional variables, and the typical time scale is

$$
t_{0}=\frac{\operatorname{LV}(0)}{k(0) A P(0)}
$$


Equation (4) then reduces to

$$
\frac{d m(t)}{d t}=-c_{1}(t) m(t)^{2}+c_{2}(t) m(t),
$$

where I have dropped the ${ }^{*}$ notation herein, and

$$
c_{1}(t)=\frac{k(t) T(t)}{V^{2}(t)}, \quad c_{2}(t)=\frac{k(t)}{V(t)} .
$$

Equation (9) governs the fundamental equation of our system, which is driven by changes in $T(t)$ and hence $c_{1}(t)$ and $c_{2}(t)$. This equation is a logistic equation, common in population biology, but with coefficients which vary slowly with time. If $c_{1}$ and $c_{2}$ were constant then the system would have an equilibrium mass

$$
m=\frac{c_{2}}{c_{1}}=\frac{V(t)}{T(t)} \equiv \gamma(t) .
$$

Thus with $c_{1}(t)$ and $c_{2}(t)$ changing with time, the mass effectively chases this changing equilibrium point, which is the solution when just the perfect gas law is applied, Equation (3). This is the solution when the permeability $k$ of the cork is infinite, that is, when there is no cork, and forms an upper bound to the air mass loss.

\section{Estimations}

In this section I find estimates of mass changes due to wine expansion and due to temperature induced air pressure changes. I take a typical temperature range of $\mathrm{T}=280 \mathrm{~K}$ to $\mathrm{T}=310 \mathrm{~K}$, appropriate for a diurnal cycle. In scaled coordinates this is a temperature change of $30 / 280=\epsilon \approx 0.11$. 
Assuming no wine expansion, so $V(t)=1$, and a temperature change of $\mathrm{T}(\mathrm{t})=1+\epsilon$, Equation (11) gives the mass change

$$
m-1=\frac{1}{1+\epsilon}-1 \approx-\epsilon .
$$

That is, the percentage mass loss due to temperature induced pressure changes in the air volume is roughly $11 \%$ for one diurnal temperature change.

The typical wine expansion coefficient is $\beta=2 \times 10^{-4} / \mathrm{K}[2,6,7]$. For a standard $750 \mathrm{ml}$ bottle of wine the volume increase over 30 degrees is reported to be in the range of $4.73-7.4 \mathrm{ml}$ compared with a head space volume in the order of 5-15 ml. Hence the upper limit of proportional mass loss due to wine expansion is of the order of $\mathbf{3 0 - 1 0 0 \%}$ and is hence very comparable to the changes due to pressure increases within the head space. This also implies that overfilled bottles, with little headspace, are particularly prone to wine expansion effects if temperate changes are large. Anecdotally, manufacturers have reported international shipments of wine where temperature changes have been large enough for the pressure to expel a third of the corks. Almost certainly this is due to insufficient headspace to allow for wine expansion.

\section{Approximate solutions}

Equation (9) is difficult to solve exactly, hence I analyse various approximate solutions and compare these for a linear temperature change. These approximations assume that the driving temperature $T(t)$ changes slowly and remains close to the initial equilibrium state.

The first approximate solution, labelled $\mathrm{S} 1$ in the figures below, is simply the upper limit $m=\gamma$, Equation (11). The second solution, labelled S2, assumes $\boldsymbol{c}_{1}(\boldsymbol{t})$ and $\boldsymbol{c}_{2}(\boldsymbol{t})$ are constant in Equation (9), so that

$$
\mathrm{m}(\mathrm{t}) \approx \frac{\gamma(\mathrm{t}) \mathrm{m}(0)}{\mathrm{m}(0)+[\gamma(\mathrm{t})-\mathrm{m}(0)] \exp \left[-\mathrm{c}_{2}(\mathrm{t}) \mathrm{t}\right]} .
$$


This solution will have errors of order $d \gamma / d t$ and $d c_{2} / d t$. As $t \rightarrow \infty$ the mass approaches the equilibrium solution $\mathrm{m} \rightarrow \gamma$.

Another approximation assumes the solution remains close to the equilibrium so that Equation (9) becomes

$$
\frac{\mathrm{dm}(\mathrm{t})}{\mathrm{dt}} \approx-\mathrm{c}_{2}(\mathrm{t})[\mathrm{m}(\mathrm{t})-\gamma(\mathrm{t})],
$$

with solution

$$
m(t) \approx m(0)+\frac{\int_{\tau=0}^{t} c_{2}(\tau) \gamma(\tau) \exp \left(\int_{0}^{\tau} c_{2}(z) d z\right) d \tau}{\exp \left(\int_{0}^{t} c_{2}(\tau) d \tau\right)} .
$$

Whilst this approximate solution can be evaluated, it is not illuminating since simple expressions for $\mathrm{m}(\mathrm{t})$ are not available for all but the most trivial temperature changes, and even numerically it is cumbersome to evaluate. As the key variables $c_{2}(t)$ and $\gamma(t)$ change it is not entirely clear how $m(t)$ changes using this expression. Thus Equation (15) does not give physical insight into how $m(t)$ changes as key parameters vary. Hence I consider a technique described by Shepherd and Stojkov [5]. The method uses two different time scales, $t_{0} \equiv t$ and $t_{1} \equiv \epsilon t$, with $\epsilon$ small and $c_{2}=c_{2}\left(t_{1}\right)$ and $\gamma=\gamma\left(t_{1}\right)$. That is, $c_{1}$ and $c_{2}$ vary on the slower time scale, while $m(t)$ chases equilibrium on a faster time scale. The mass is written as $m=m\left(t_{0}, t_{1}, \epsilon\right)$ with Equation (14) becoming

$$
\frac{\partial m}{\partial t_{0}}+\epsilon \frac{\partial m}{\partial t_{1}}=-c_{2}\left(t_{1}\right)\left[m-\gamma\left(t_{1}\right)\right] .
$$

Expanding

$$
m=m_{0}\left(t_{0}, t_{1}\right)+\epsilon m_{1}\left(t_{0}, t_{1}\right)+\cdots
$$

and equating coefficients gives

$$
\frac{\partial m_{0}}{\partial t_{0}}=-c_{2}\left(t_{1}\right)\left[m-\gamma\left(t_{1}\right)\right],
$$




$$
\frac{\partial m_{1}}{\partial t_{0}}=-c_{2}\left(t_{1}\right) m_{1}-\frac{\partial m_{0}}{\partial t_{1}} .
$$

These have solutions, using the initial condition $m=m_{a}(0)$,

$$
\begin{aligned}
& m_{0}=(m(0)-\gamma) e^{-c_{2} t_{0}}+\gamma, \\
& m_{1}=\left(\gamma^{\prime} t+\frac{1}{2}(m(0)-\gamma) c_{2}^{\prime} t^{2}-\frac{\gamma^{\prime}}{c_{2}} e^{c_{2} t}+\frac{\gamma^{\prime}}{c_{2}}\right) e^{-c_{2} t_{0}},
\end{aligned}
$$

where $\gamma$ and $c_{2}$ are functions of $t_{1}$ and hence $\gamma^{\prime}=d \gamma / d t_{1}$. Equations (17), (20) and (21) represent my third solution, labelled S3.

This third solution has the long term behaviour

$$
\mathrm{m}(\mathrm{t}) \approx \gamma(\epsilon \mathrm{t})-\epsilon \frac{\gamma^{\prime}(\epsilon \mathrm{t})}{\mathrm{c}_{2}(\epsilon \mathrm{t})} .
$$

That is, the mass attempts to reach the equilibrium point $\gamma$ but since this equilibrium keeps changing slowly with time, it lags by a small amount. In the resultant figures I label this result, Equation (22), as S4.

\section{$5 \quad$ Results}

This section considers the numerical and approximate solutions for a simple linear temperature change, in scaled coordinates, of $\mathrm{T}=\epsilon \mathrm{t}$, for $\mathrm{t}>0$ with $T=1$ for $t \leq 0$. All results shown here use $\epsilon=0.1$. These solutions are scaled and hence irrespective of absolute temperature and pressures. However, inclusion of wine expansion requires some volume dimensions. I consider a $750 \mathrm{ml}$ bottle of wine, a $4 \mathrm{~cm}$ long headspace of radius $1 \mathrm{~cm}, \beta=2 \times 10^{-4} / \mathrm{K}$ as the volumetric expansion coefficient, and an initial temperature of $10^{\circ} \mathrm{C}$.

The numerical solutions to Equation (9) was done using a standard first order Euler time step $m(t+d t) \approx m(t)+d t \frac{d m}{d t}$. The routine was tested 


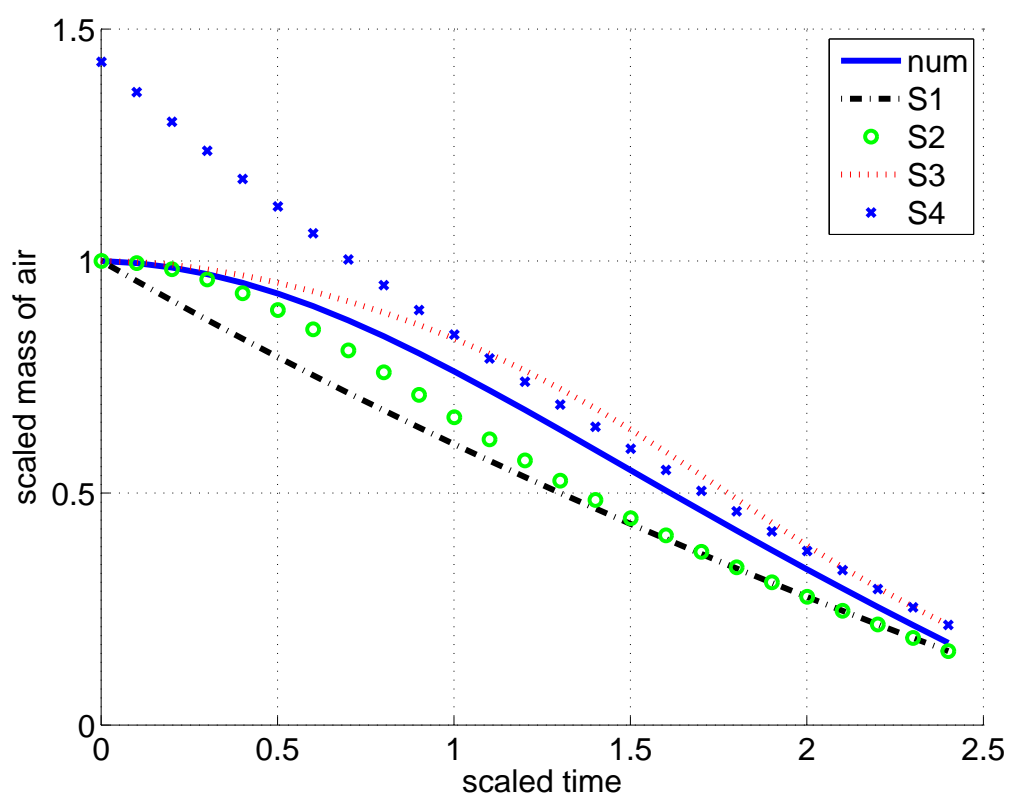

FiguRE 2: Air mass versus time for a linearly changing temperature with wine volume increasing. Results correspond to different solution types.

for convergence and found to be $\mathrm{O}(\mathrm{dt})$ accurate as expected. A value of $\mathrm{dt}=0.01$, for a range of $t \in[0,2.5]$ was used giving solutions to within $0.01 \%$ accuracy.

Figure 2 shows the scaled mass of air changing as a function of time. The various solutions shown are: the numerical solution to Equation (9), labelled 'num'; the fully permeable solution, Equation (11) -labelled S1; the approximation with $\mathbf{c}_{1}$ and $\boldsymbol{c}_{2}$ assumed constant, Equation (13) - S2; the perturbation solution, Equation (17) - S3; and the asymptotic solution, Equation (22) - S4. These results illustrate that the numerical solution is bounded between the asymptotic limits given by $\mathrm{S} 4$ and the freely permeable cork solution S1. 


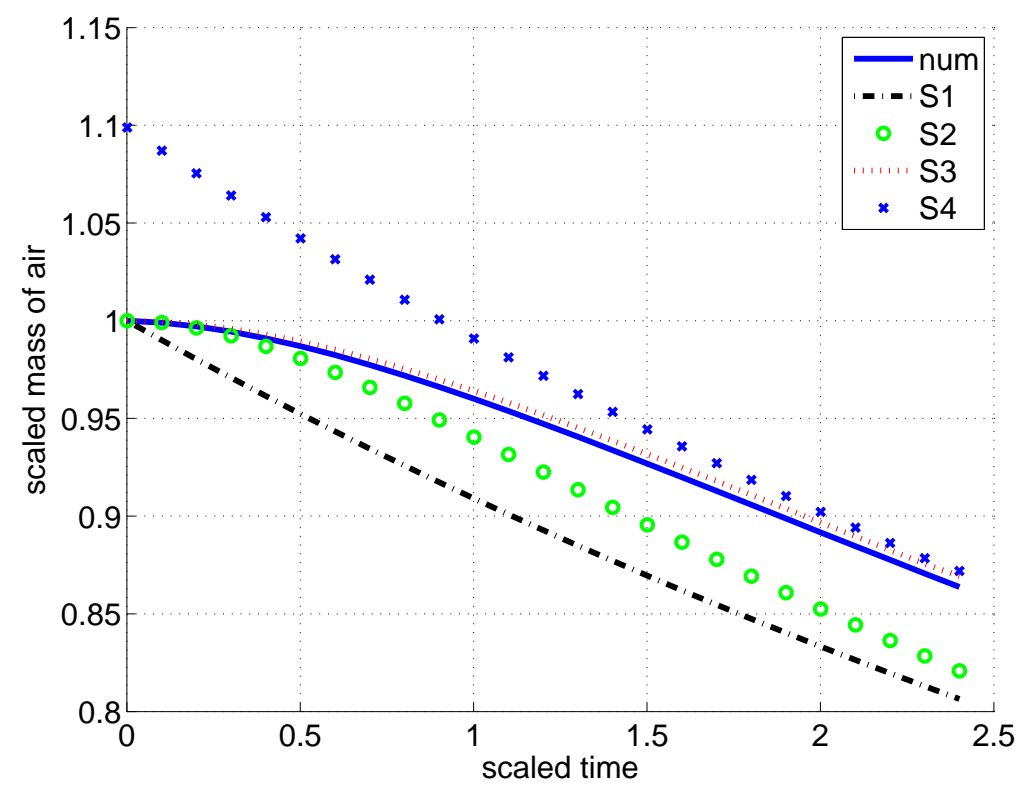

Figure 3: Air mass versus time for a linearly changing temperature with wine volume constant. Results correspond to different solution types.

I note that the behaviour shown here is slightly different to that illustrated by Mercer et al. [4] which took the simplified case of constant air volume. These results are recalculated in Figure 3 which better illustrates how the numerical solution approaches the limiting solutions $\gamma$ with a time lag dependence. This would be the situation where the headspace volume was large enough that volumetric expansion effects of the wine was unimportant.

In Figures 4 and 5 the pressure within the head space is modelled for both of the varying volume and constant volume cases discussed in Figures 2 and 3. For Figure 5 where volume is held constant, the various strengths and behaviour of the models are clear with the numerical solution approaching the asymptotic solution S4 and being well matched by the approximation 


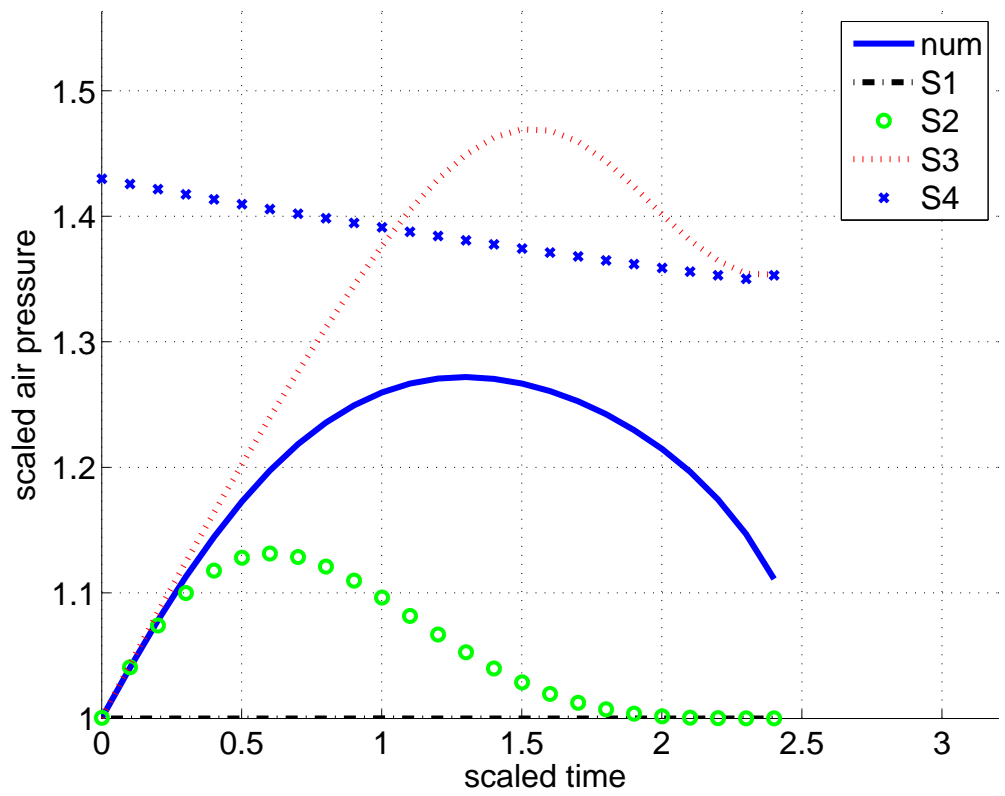

Figure 4: Air pressure within the headspace as a function of time with wine expansion. Results correspond to different solution types.

S3. In Figure 4 the asymptotic solutions do not match the pressure results, except for small times when the approximation assumptions of being close to equilibrium are valid. In these scenarios the increase in wine volume is reducing the air volume to zero, making the dynamics very complicated.

\section{Conclusions}

I have explored a simple model of temperature effects on air flow through a wine cork. By coupling basic physical laws, a logistic type equation is recovered with time varying coefficients. When volumetric expansion of the wine is minimal then various asymptotic solutions can be shown to approximate 


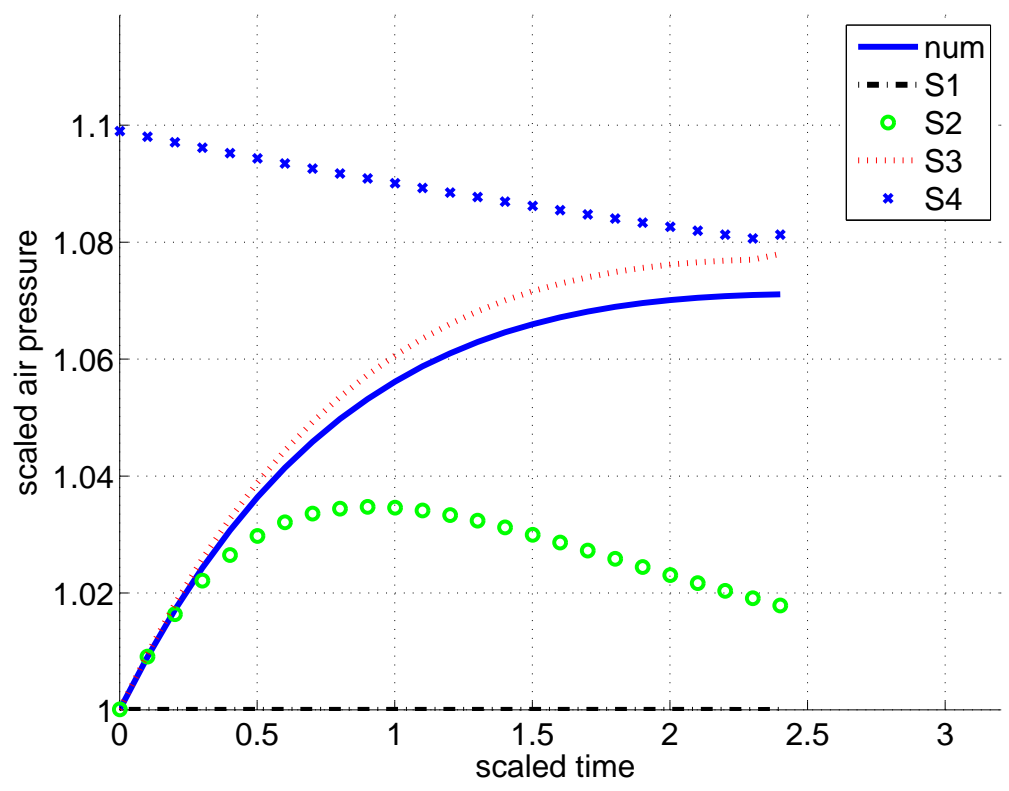

FIgURE 5: Air pressure within the headspace as a function of time with no wine expansion. Results correspond to different solution types. 
the amount of air mass left in the head space. In particular, these show how the solution tends towards the changing equilibrium state with a time lag dependent on the rate of equilibrium change, Equation (22). However, in cases where wine expansion is a dominant effect, the full numerical solution is necessary.

This study is not designed as an extensive analysis of this problem, and there are many aspects that warrant further investigation. These include allowing for other temperature dependent effects such as vapour pressures, thermodynamics, solubility and air density changes. These are the subject of future research.

\section{References}

[1] J. Bear, Dynamics of fluids in porous media, Dover, New York, 1972. C447

[2] R. B. Boulton, V. L. Singleton, L. F. Bisson, R. E. Kunkee, Principles and practices of winemaking, Springer, New York, 1998. C448, C450

[3] P. L. Laffer, Management of wine quality during transport. Proceedings of the twelfth Australian wine industry technical conference, ed. R Blair, P Williams and S Pretorius, 219-221, 2004. C445

[4] G. N. Mercer, A. Wilkins, J. Crook, S. I. Barry, A. Fowler, The shelf life of wine, Proceedings MISG 2008, ed: T. R. Marchant, M. Edwards and G. N. Mercer, Univ. Wollongong, 2008. C445, C454

[5] J. J. Shepherd, L. Stojkov, The logistic population model with slowly varying carrying capacity, ANZIAM J(E) (EMAC2005), 47, C492-C506, 2007. http://anziamj .austms.org.au/ojs/index.php/ ANZIAMJ/article/view/1058 C451 
[6] J. Tremblay, L'influence de la tempurature sur le transport des vins par containers. Rev. Fr. Oenol., 24, 11-18, 1984. C450

[7] C. Vannobel, Changes in bottled wine volumes, Rev. Fr. Oenol., 125, 41-46, 1990. C450

\section{Author address}

1. Steven I. Barry, University of New south Wales @ ADFA, Canberra, Australia.

mailto:s.barry@adfa.edu.au 